\title{
Existence and Approximation of Manifolds for the Swift-Hohenberg Equation with a Parameter
}

\author{
Yanfeng Guo $\mathbb{D}^{1,2}$ Chunxiao Guo $\mathbb{D}^{3},{ }^{3}$ and Donglong Li ${ }^{1}$ \\ ${ }^{1}$ School of Science, Guangxi University of Science and Technology, Liuzhou, Guangxi 545006, China \\ ${ }^{2}$ School of Mathematics and Physics, China University of Geosciences, Wuhan, Hubei 430074, China \\ ${ }^{3}$ Department of Mathematics, China University of Mining and Technology Beijing 100083, China
}

Correspondence should be addressed to Yanfeng Guo; guoyan_feng@aliyun.com

Received 28 February 2018; Revised 21 May 2018; Accepted 30 May 2018; Published 12 July 2018

Academic Editor: Francisco R. Villatoro

Copyright (C) 2018 Yanfeng Guo et al. This is an open access article distributed under the Creative Commons Attribution License, which permits unrestricted use, distribution, and reproduction in any medium, provided the original work is properly cited.

\begin{abstract}
The existence and approximation of manifolds for the Swift-Hohenberg equation with a proper parameter have mainly been studied. Using the backward-forward systems from Swift-Hohenberg equation, the existence and specific representation forms of manifolds for Swift-Hohenberg equation with a parameter have been obtained. Meanwhile, we make use of technique of deposition of lower and higher frequency spaces of solutions and assume the reduced system to obtain the main numeration approximation system of approximation solution for the original system Swift-Hohenberg equation with a proper parameter.
\end{abstract}

\section{Introduction}

Recently, many mathematicians and physicians are interested in manifolds of some partial differential equations (PDEs). Recently, various approximations of manifolds of PDEs have been proposed, for example, amplitude equations approaches and so on [1-5].

In this paper, we have studied the manifolds of the real Swift-Hohenberg equation

$$
u_{t}=\lambda u-\left(1+\partial_{x x}\right)^{2} u-u^{3}
$$

with a proper parameter $\lambda$, by which transverse pattern formations in both degenerate optical parametric oscillation and degenerate four-wave mixing are shown in the limit of small signal detuning [6].

It is well known that the Swift-Hohenberg model was proposed to describe pattern formation in convection by Swift and Hohenberg in studying the convective instability in the Rayleigh-Bénard convection [7]. However, this mode does not adequately explain this phenomenon. Then some generalizations of this model have been derived in various branches in [8-12], such as nonlinear optics for lasers, magnetoconvection, and biological, chemical, and liquidcrystal light-valve experiment. In addition, the complex
Swift-Hohenberg equation has been derived in $[13,14]$. And it is very important to consider properties of real SwiftHohenberg equation in some branches, such as physics, hydrodynamics, and nonlinear optics; see $[15,16]$.

Many dynamical behaviors for the local and nonlocal one-dimensional Swift-Hohenberg equation, such as attractors and invariant manifolds, have been investigated in [1721]. We know that some manifolds for partial differential equations are important to study the dynamical behaviors for them, which include stable and unstable manifolds [1,22] and center manifolds $[23,24]$. Some results about stable and unstable manifolds for Swift-Hohenberg equation have been obtained in [1]. However, there have been a few results about approximations of manifolds for Swift-Hohenberg equation with a proper parameter until now. Particularly, the numeration approximation system and some numerical solutions for Swift-Hohenberg equation with a proper parameter have not been considered by few authors. Here, approximation of manifolds has been mainly investigated by technique of deposition of solution between lower and higher frequency spaces. And we have used the numeration approximation system to solve the approximation solutions. This idea has been considered a few times to approximate manifolds for some system, especially for Swift-Hohenberg equation. Here, 
we will give the main idea of this method in the following process.

In this paper, the existence and approximation of manifolds for the real Swift-Hohenberg equation

$$
u_{t}=\lambda u-\left(1+\partial_{x x}\right)^{2} u-u^{3}
$$

with a proper parameter have been investigated by the idea in [5]. On the basis of existence of parameter manifold, the numeration approximation systems of iterative levels have been given by backward-forward systems. Then, some new results have been obtained. The results in this manuscript have been used to obtain the approximation solution of some real partial differential equations. This work can be extended to some real systems in a larger field of math. Firstly, the existence and the representation form of manifolds for Swift-Hohenberg equation with a proper parameters are given. Secondly, the numeration approximation system is considered based on manifolds with a proper parameter using deposition of solutions between lower and higher frequency spaces. Finally, we have obtained the corresponding numeration approximation system for numerical solutions of SwiftHohenberg equation with proper parameters under some conditions.

\section{Preliminaries}

The work spaces are mainly given by the Hilbert spaces $H_{1}=D(A)$ and $H=L^{2}(0, \pi)$, where $A=(1+\Delta)^{2}$ and $H_{1}$ is compactly and densely embedded in $H$. The operator $A: H_{1} \longrightarrow H$ is linear and the spectrum of $-A$ satisfies $\sigma(-A)<0$. In addition, the interpolated space $H_{\alpha}$ is a space between $H_{1}$ and $H$ with $\alpha \in[0,1)$. Let $L_{\lambda}=-A+\lambda$ be linear operators including a simple parameter. Here $L_{\lambda}$ map $H_{\alpha}$ into $H$ and depend continuously on $\lambda$.

For the convenience of studying, the Swift-Hohenberg equation has been rewritten in the following form:

$$
d u=\left(\lambda u-\left(1+\partial_{x x}\right)^{2} u-u^{3}\right) d t=\left(L_{\lambda} u+F(u)\right) d t
$$

with initial condition $u(0, x)=u_{0}(x) \in H_{\alpha}$ and Dirichlet boundary conditions $u(t, 0)=u(t, \pi)=0$ on $D=[0, \pi]$, where $F(u)$ is defined as $F(u)=-u^{3}$, which is a trilinear and continuous function from $H_{\alpha} \times H_{\alpha} \times H_{\alpha}$ to $H, \alpha \in[0,1)$. Here, we define $F\left(u_{1}, u_{2}, u_{3}\right)=-u_{1} u_{2} u_{3}$. The operator $A=(1+$ $\Delta)^{2}$ is closed self-adjoint linear operator with dense domain $D(A)=H_{1}=H^{4}(0, \pi) \cap H_{0}^{2}(0, \pi)$. In addition, $L_{\lambda}$ is selfadjoint operator with an orthonormal basis of eigenfunctions $\left\{e_{k}=\sqrt{2 / \pi} \sin (k x)\right\}_{k \in \mathbb{N}}$ in $H$ and corresponding eigenvalues $\left\{\beta_{k}(\lambda)=\lambda-\left(1-k^{2}\right)^{2}\right\}_{k \in \mathbb{N}}$.

Assume that $H^{c}=\operatorname{span}\left\{e_{1}, e_{2}, \ldots, e_{m}\right\}$, where $m$ is finite. Let the topological complement of $H^{c}$ be $H_{\alpha}^{s}$ and the function $h_{p m}$ maps $H^{c}$ to $H_{\alpha}^{s}$. Then we have the high frequency part $u_{s}(t)=P_{s} u(t)$ and low frequency part $u_{c}(t)=P_{c} u(t)$, where $P_{c}: H \longrightarrow H^{c}$ and $P_{s}=I-P_{c}$. Thus, $u=P_{c} u+P_{s} u=u_{c}+u_{s}$ for any $u \in L^{2}(0, \pi)$.

\section{Approximation of Parameter Manifolds}

According to the approximation methods in [5], using backward-forward systems of Swift-Hohenberg equation and $u=P_{c} u+P_{s} u=u_{c}+u_{s}$, we assume the following reduced system:

$$
\begin{aligned}
d u_{c}^{(1)} & =L_{\lambda}^{c} u_{c}^{(1)} d t, \\
u_{c}^{(1)}(0, x) & =\xi, \\
\xi \in H^{c}, t \in[-T, 0], & \\
d u_{s}^{(1)} & =\left[L_{\lambda}^{s} u_{s}^{(1)}+P_{s} F\left(u_{c}^{(1)}(t-T)\right)\right] d t, \\
u_{s}^{(1)}(0) & =0,
\end{aligned}
$$

$$
t \in[0, T],
$$

where $L_{\lambda}^{c}:=P_{c} L_{\lambda}, L_{\lambda}^{s}:=P_{s} L_{\lambda}, u^{(1)}=u_{c}^{(1)}+u_{s}^{(1)}$, and $\xi \in H^{c}$. Equation (4) is the linear part of (3) in space $H_{c}$, which is assumed and seen as the first iteration level in $H_{c}$ of reduced system (4)-(5). From the above system, the existence and specific presentation forms of manifolds under parameter $\lambda$ are given by the following theorem with the initial values of $u_{c}^{(1)}$ and $u_{s}^{(1)}$.

Theorem 1. Consider the Swift-Hohenberg equation (3), where $F$ is a trilinear function. Assume also that $\beta_{j}(\lambda)<0$ for all $j>$ $m$. Let $\mathscr{I}=\{1, \ldots, m\}, m=\operatorname{dim}\left(H^{c}\right)$. If, for all $\left(k_{1}, k_{2}, k_{3}\right) \in$ $\mathscr{I}^{3},<F\left(e_{k_{1}}, e_{k_{2}}, e_{k_{3}}\right), e_{j}>\neq 0$ when $\beta_{k_{1}}+\beta_{k_{2}}+\beta_{k_{3}}-\beta_{j}>0$, $j>m$, then the pullback limit for the solution of (5) exists and is given by

$$
\begin{aligned}
h_{\lambda}^{(1)}(\xi) & =\lim _{T \longrightarrow+\infty} u_{s}^{(1)}(T, x) \\
& =\int_{-\infty}^{0} e^{-t L_{\lambda}^{s}} P_{s} F\left(u_{c}^{(1)}(t, \xi)\right) d t, \quad \forall \xi \in H^{c},
\end{aligned}
$$

where $u_{c}^{(1)}(t)$ is the solution of (4) and

$$
u_{c}^{(1)}(t)=e^{t L_{\lambda}^{c}} \xi
$$

Moreover, $h_{\lambda}^{(1)}(\xi)$ has the following analytic expression:

$$
\begin{gathered}
h_{\lambda}^{(1)}(\xi)=-\sum_{j=m+1}^{\infty} \sum_{k_{1}, k_{2}, k_{3}=1}^{m} \frac{1}{\beta_{k_{1}}+\beta_{k_{2}}+\beta_{k_{3}}-\beta_{j}} \xi_{k_{1}} \xi_{k_{2}} \xi_{k_{3}} \\
\cdot\left\langle P_{s}\left(e_{k_{1}} e_{k_{2}} e_{k_{3}}\right), e_{j}\right\rangle e_{j}, \quad \text { for } j>m,
\end{gathered}
$$

where $\xi_{k}=<\xi, e_{k}>, k=1, \ldots, m$.

Proof. It is easy to obtain the solution of (4) given by (7). By using the variation-of-constants formula, we can formally obtain the solution of (5), which is given by

$$
u_{s}^{(1)}(T, x)=\int_{-T}^{0} e^{-t L_{\lambda}^{s}} P_{s} F\left(u_{c}^{(1)}(t)\right) d t,
$$

where $u_{c}^{(1)}(t)$ is the solution of (4) and has the form of (7). According to the conditions, we can obtain that limit of (9) exists. Plugging (7) into (9), we give the representation as (8). 


\section{Approximation of Solution Based Reduced System}

Assume that the subspace $H^{c}=\operatorname{span}\left\{e_{1}, e_{2}\right\}$ and take $9<$ $\lambda \leq 64$. Now, the numeration system of approximation of solution is investigated using manifold under proper parameter by parts of solution in lower frequency spaces according to the reduced system of (3). In order to investigate the approximation of solution, the derivation process of an approximation system is given as follows.

In order to replace the nonlinear term with the pullback limit of solution in high-frequency spaces, we consider the system

$$
\begin{aligned}
d u_{c}^{(1)} & =L_{\lambda}^{c} u_{c}^{(1)} d t, \\
u_{c}^{(1)}(t, x) & =\xi(t), \\
\xi(t) \in H^{c}, t \in[t-T, t], & \\
d u_{s}^{(1)} & =\left[L_{\lambda}^{s} u_{s}^{(1)}+P_{s} F\left(u_{c}^{(1)}(t-T)\right)\right] d t, \\
u_{s}^{(1)}(t, x) & =0,
\end{aligned}
$$

$$
t \in[t, t+T]
$$

where $L_{\lambda}^{c}:=P_{c} L_{\lambda}, L_{\lambda}^{s}:=P_{s} L_{\lambda}$, and

$$
\begin{aligned}
\xi(t) & =\left\langle\xi(t), e_{1}\right\rangle e_{1}+\left\langle\xi(t), e_{2}\right\rangle e_{2} \\
& =\xi_{1}(t) e_{1}+\xi_{2}(t) e_{2} \in H^{c} .
\end{aligned}
$$

Similar to the process in Section 2, there is a pullback limit of solution for the above system (10)-(11) written as $h_{\lambda}^{(1)}(\xi(t))$.

Then projecting (3) into the subspace $H^{c}$, we have

$$
d u_{c}=\left(L_{\lambda}^{c} u_{c}+P_{c} F\left(u_{c}+u_{s}\right)\right) d t
$$

where $u_{c}=P_{c} u$ and $u_{s}=P_{s} u$, with $P_{c}$ and $P_{s}$ being the canonical projectors associated with $H^{c}$ and $H^{s}$, respectively.

Considering $u_{s}(t)=P_{s} u(t)$ and substituting the pullback $\operatorname{limit} h_{\lambda}^{(1)}(\xi(t))$ of the first lever system (10)-(11) into the above equation, we obtain reduced equation,

$$
d \xi(t)=\left(L_{\lambda}^{c} \xi+P_{c} F\left(\xi(t)+h_{\lambda}^{(1)}(\xi(t))\right)\right) d t,
$$

which provides an approximation solution of the SwiftHohenberg equation projected onto the low-frequency parts.

From (8), we know that the coefficients of $\left\{e_{k}(x)\right\}$ contained in the expansion of $h_{\lambda}^{(1)}$ are decayed. Therefore, the analytic representation of $h_{\lambda}^{(1)}$ can be given from (8); the nonlinear term $F_{k_{1}, k_{2}, k_{3}}^{j}=<F\left(e_{k_{1}}, e_{k_{2}}, e_{k_{3}}\right), e_{j}>$ is given by $-<e_{k_{1}} e_{k_{2}} e_{k_{3}}, e_{j}>$ such that

$$
\begin{aligned}
& F_{k_{1} k_{2} k_{3}}^{j}=\frac{1}{2 \pi} \text { for } k_{1}+k_{2}+k_{3}=j, \\
& F_{k_{1} k_{2} k_{3}}^{j}=-\frac{1}{2 \pi}
\end{aligned}
$$

for $k_{1}+k_{2}-k_{3}=j$ or $k_{1}-k_{2}+k_{3}=j$ or $-k_{1}+k_{2}+k_{3}=j$, and

$$
F_{k_{1} k_{2} k_{3}}^{j}=0 \text { for } j \geq 7
$$

Then we can obtain the result as follows:

$$
\begin{aligned}
& h_{\lambda}^{(1)}(\xi) \\
& =-\sum_{j=3}^{\infty} \sum_{k_{1}, k_{2}, k_{3}=1}^{2} \frac{1}{\beta_{k_{1}}+\beta_{k_{2}}+\beta_{k_{3}}-\beta_{j}} \xi_{k_{1}} \xi_{k_{2}} \xi_{k_{3}}\left\langle P_{s}\left(e_{k_{1}} e_{k_{2}} e_{k_{3}}\right),\right. \\
& \left.\quad e_{j}\right\rangle e_{j}=-\frac{1}{2 \pi} D_{3}^{(111)} \xi_{1}^{3} e_{3}-\frac{3}{2 \pi} D_{3}^{(122)} \xi_{1} \xi_{2}^{2} e_{3}+\frac{3}{2 \pi} D_{4}^{(112)} \xi_{1}^{2} \xi_{2} e_{4} \\
& \quad+\frac{3}{2 \pi} D_{4}^{(122)} \xi_{1} \xi_{2}^{2} e_{5}+\frac{1}{2 \pi} D_{6}^{(222)} \xi_{2}^{3} e_{6}
\end{aligned}
$$

where $\xi_{1}=<\xi, e_{1}>, \xi_{2}=<\xi, e_{2}>$, and $D_{j}^{\left(k_{1} k_{2} k_{3}\right)}=$ $1 /\left(\beta_{k_{1}}+\beta_{k_{2}}+\beta_{k_{3}}-\beta_{j}\right)$. Similar to the above processing, the analytic formula of $h_{\lambda}^{(1)}(\xi(t))$ can be given, which has a complex formula in form. Then, there are some difficulties by using directly the analytic formula of $h_{\lambda}^{(1)}(\xi(t))$ to obtain the vector $P_{c} F\left(\xi(t)+h_{\lambda}^{(1)}(\xi(t))\right)$ when $\xi(t)$ varies in $H^{c}$ in spite of any case. So, replacing $u_{s}^{(1)}[\xi(t)](t+T)$ with $h_{\lambda}^{(1)}(\xi(t))$ when initial condition is $\xi(t)$ at $t$, where $u_{s}^{(1)}$ is obtained by the above backward-forward system (4)-(5), we consider the following substitutive reduced equation:

$$
\begin{aligned}
& d \xi(t) \\
& \quad=\left(L_{\lambda}^{c} \xi(t)+P_{c} F\left(\xi(t)+u_{s}^{(1)}[\xi(t)](t+T)\right)\right) d t, \\
& \xi(0)=\phi, \\
& t>0,
\end{aligned}
$$

where $\phi$ is the initial datum; $u_{s}^{(1)}[\xi(t)]$ is given by the following system:

$$
\begin{aligned}
d u_{c}^{(1)} & =L_{\lambda}^{c} u_{c}^{(1)} d s, \\
\left.u_{c}^{(1)}(s)\right|_{s=t} & =\xi(t), \\
s \in[t-T, t], & \\
d u_{s}^{(1)} & =\left(L_{\lambda}^{s} u_{s}^{(1)}+P_{s} F\left(u_{c}^{(1)}(s-T)\right)\right) d s, \\
\left.u_{s}^{(1)}(s)\right|_{s=t} & =0,
\end{aligned}
$$

$$
s \in[t, t+T]
$$

Now, we assume that

$$
\begin{aligned}
\xi(t, x) & =\xi_{1}(t) e_{1}(x)+\xi_{2}(t) e_{2}(x) \\
& =y_{1}^{(1)}(t) e_{1}(x)+y_{2}^{(1)}(t) e_{2}(x)=u_{c}^{(1)}(t, x) .
\end{aligned}
$$


Then, when $N \geq 7$ and $t \in[t, t+T]$, taking inner product with $e_{1}$ and $e_{2}$ on both sides of (18), respectively, we obtain that

$$
\begin{aligned}
& d \xi_{1}(t)=\beta_{1}(\lambda) \xi_{1}(t)-\frac{3}{2 \pi}\left(\xi_{1}^{3}+2 \xi_{1} \xi_{2}^{2}\right. \\
& \left.-\left(\xi_{1}^{2}-\xi_{2}^{2}\right) y_{3}^{(1)}-2 \xi_{1} \xi_{2} y_{4}^{(1)}-\xi_{2}^{2} y_{5}^{(1)}\right)+\frac{3}{\pi} \\
& \cdot \xi_{1} \sum_{i=3}^{N}\left(y_{i}^{(1)} y_{i+2}^{(1)}-\left[y_{i}^{(1)}\right]^{2}\right)+\frac{3}{\pi} \\
& \cdot \xi_{2} \sum_{i=3}^{N}\left(y_{i}^{(1)} y_{i+3}^{(1)}-y_{i}^{(1)} y_{i+1}^{(1)}\right) \\
& +\frac{1}{2 \pi}\left(\sum_{i=3}^{N} \sum_{j=i+4}^{N} y_{i}^{(1)} y_{j}^{(1)} y_{j-i-1}^{(1)}\right. \\
& \left.-\sum_{i=3}^{N} \sum_{j=i+2}^{N} y_{i}^{(1)} y_{j}^{(1)} y_{j-i+1}^{(1)}\right)+\frac{1}{\pi} \\
& \cdot \sum_{i=3}^{N} \sum_{j=3}^{N}\left(y_{i}^{(1)} y_{j}^{(1)} y_{i+j+1}^{(1)}-y_{i}^{(1)} y_{j}^{(1)} y_{i+j-1}^{(1)}\right) d t, \quad t>0, \\
& d \xi_{2}(t)=\beta_{2}(\lambda) \xi_{1}(t)-\frac{3}{2 \pi}\left(\xi_{2}^{3}+2 \xi_{1}^{2} \xi_{2}-\xi_{1}^{2} y_{4}^{(1)}\right. \\
& \left.-2 \xi_{1} \xi_{2}\left(y_{5}^{(1)}-y_{3}^{(1)}\right)-\xi_{2}^{2} y_{6}^{(1)}\right)+\frac{3}{\pi} \\
& \cdot \xi_{1} \sum_{i=3}^{N}\left(y_{i}^{(1)} y_{i+3}^{(1)}-y_{i}^{(1)} y_{i+1}^{(1)}\right)+\frac{3}{\pi} \\
& \cdot \xi_{2} \sum_{i=3}^{N}\left(y_{i}^{(1)} y_{i+4}^{(1)}-\left[y_{i}^{(1)}\right]^{2}\right) \\
& +\frac{1}{2 \pi}\left(-\sum_{i=3}^{N} \sum_{j=i+1}^{N} y_{i}^{(1)} y_{j}^{(1)} y_{j-i+2}^{(1)}\right. \\
& \left.+\sum_{i=3}^{N} \sum_{j=i+5}^{N} y_{i}^{(1)} y_{j}^{(1)} y_{j-i-2}^{(1)}\right)+\frac{1}{2 \pi} \\
& \cdot \sum_{i=3}^{N} \sum_{j=3}^{N}\left(y_{i}^{(1)} y_{j}^{(1)} y_{i+j+2}^{(1)}-y_{i}^{(1)} y_{j}^{(1)} y_{i+j-2}^{(1)}\right) d t, \quad t>0,
\end{aligned}
$$

with $\xi_{1}(0)=<\phi, e_{1}>$ and $\xi_{2}(0)=<\phi, e_{2}>$, where $\xi_{t}=$ $\xi_{1}(t) e_{1}+\xi_{2}(t) e_{2}$ and $y_{j}^{(1)}, j=3, \ldots$, given via the following system:

$$
\begin{aligned}
& d y_{1}^{(1)}(s)=\beta_{1}(\lambda) y_{1}^{(1)}(s) d s, \quad s \in[t-T, t] \\
& d y_{2}^{(1)}(s)=\beta_{2}(\lambda) y_{2}^{(1)}(s) d s, \quad s \in[t-T, t]
\end{aligned}
$$

$$
\begin{aligned}
& d y_{3}^{(1)}(s)=\left(\beta_{3}(\lambda) y_{3}^{(1)}(s)+\frac{1}{2 \pi}\left(\left[y_{1}^{(1)}(s-T)\right]^{3}\right.\right. \\
& \left.\left.-3 y_{1}^{(1)}(s-T)\left[y_{2}^{(1)}(s-T)\right]^{2}\right)\right) d s, \\
& s \in[t, t+T] ; \\
& d y_{4}^{(1)}(s)=\left(\beta_{4}(\lambda) y_{4}^{(1)}(s)+\frac{3}{2 \pi}\left[y_{1}^{(1)}(s-T)\right]^{2} y_{2}^{(1)}(s\right. \\
& -T)) d s, \quad s \in[t, t+T] ; \\
& d y_{5}^{(1)}(s)=\left(\beta_{5}(\lambda) y_{5}^{(1)}(s)+\frac{3}{2 \pi} y_{1}^{(1)}(s-T)\right. \\
& \left.\cdot\left[y_{2}^{(1)}(s-T)\right]^{2}\right) d s, \quad s \in[t, t+T] ; \\
& d y_{6}^{(1)}(s)=\left(\beta_{6}(\lambda) y_{6}^{(1)}(s)+\frac{1}{2 \pi}\left[y_{2}^{(1)}(s-T)\right]^{3}\right) d s, \\
& d y_{j}^{(1)}(s)=\beta_{j}(\lambda) y_{j}^{(1)}(s) d s, \\
& \left.d y_{j}^{(1)}=0, \quad s \in[t, t+t+T] ;, j=N+T\right], j=7, \ldots, N ;
\end{aligned}
$$

with $\left.y_{1}^{(1)}(s)\right|_{s=t}=\xi_{1}(t),\left.y_{2}^{(1)}(s)\right|_{s=t}=\xi_{2}(t)$, and $\left.y_{j}^{(1)}(s)\right|_{s=t}=0$, $j=3, \ldots$. That is to say,

$$
\begin{aligned}
& y_{1}^{(1)}(s)=e^{\beta_{1}(s-t)} \xi_{1}(t), \\
& y_{2}^{(1)}(s)=e^{\beta_{2}(s-t)} \xi_{2}(t), \\
& y_{3}^{(1)}(s)=\frac{\xi_{1}^{3}}{2 \pi\left(3 \beta_{1}-\beta_{2}\right)}\left[e^{3 \beta_{1}(s-T-t)}-e^{-3 \beta_{1} T}\right] \\
&- \frac{3 \xi_{1} \xi_{2}^{2}}{2 \pi\left(\beta_{1}+2 \beta_{2}-\beta_{3}\right)}\left[e^{\left(\beta_{1}+2 \beta_{2}\right)(s-T-t)}\right. \\
&-\left.e^{-\left(\beta_{1}+2 \beta_{2}\right) T}\right], \\
& y_{4}^{(1)}(s)=\frac{3 \xi_{1}^{2} \xi_{2}}{2 \pi\left(3 \beta_{1}+\beta_{2}-\beta_{4}\right)}\left[e^{\left(2 \beta_{1}+\beta_{2}\right)(s-T-t)}\right. \\
&-\left.e^{-\left(2 \beta_{1}+\beta_{2}\right) T}\right], \\
& y_{5}^{(1)}(s)=\frac{3 \xi_{1} \xi_{2}^{2}}{2 \pi\left(2 \beta_{2}+\beta_{1}-\beta_{5}\right)}\left[e^{\left(2 \beta_{2}+\beta_{1}\right)(s-T-t)}\right. \\
&-\left.e^{-\left(2 \beta_{2}+\beta_{1}\right) T}\right], \\
& y_{6}^{(1)}(s)=\frac{\xi_{2}^{3}}{2 \pi}\left[e^{3 \beta_{3}(s-T-t)}-e^{-3 \beta_{3} T}\right], \\
& y_{j}^{(1)}=0, \quad j=7, \ldots .
\end{aligned}
$$


From the above process, the numeration approximation system of solutions on finite dimensional space $H^{c}$ is given according to manifold under the proper parameter for SwiftHohenberg equation, which can be done by computer to numerate it based on this reduced system according to above idea. It is important and beneficial in some points of view to obtain partial dynamics in approximation sense on the $H^{c}$ modes in modeling of partial differential equations in practice. In fact, it is possible to achieve good modeling performances of solution from these results.

\section{Conclusion}

Therefore, existence of manifolds under proper parameters can be given for solution of some nonlinear partial differential equations. Furthermore, we have obtained the numeration approximation systems of iterative levels for solution on the basis of existence of parameter manifolds. However, the mathematical proofs in this manuscript maybe hold for the complex Swift-Hohenberg equation, which need us to further consider how to give some corresponding concepts in complex spaces. Although it is important to use the numerical solutions for some nonlinear real partial differential equations to model some practical problems according to the approximation solution in this paper, this idea can be extensively applied to solve other real partial differential equations.

\section{Data Availability}

No data were used to support this study.

\section{Conflicts of Interest}

The authors declare that they have no conflicts of interest.

\section{Authors' Contributions}

The authors contributed equally and significantly in writing this paper. All authors read and approved the final manuscript.

\section{Acknowledgments}

This research was supported by the National Natural Science Foundation of China (no. 11771444); Guangxi Natural Science Foundation (no. 2017GXNSFAA198221); Promotion of the Basic Capacity of Middle and Young Teachers in Guangxi Universities (no. 2017KY0340); the Fundamental Research Funds for the Central Universities, China University of Geosciences (Wuhan) (no. 2018061); Key Laboratory Open Fund Project of the Simulation of Manufacturing Systems and Informational Enterprise (no. 2016ZD008); Yue Qi Young Scholar Project, China University of Mining and Technology (Beijing); and CSC.

\section{References}

[1] D. Blömker, M. Hairer, and G. A. Pavliotis, "Stochastic SwiftHohenberg equaion near a change of stability," Proceedings of Equadiff, vol. 11, pp. 27-37, 2005.

[2] D. Blömker and W. Wang, "Qualitative properties of local random invariant manifolds for SPDEs with quadratic nonlinearity," Journal of Dynamics and Differential Equations, vol. 22, no. 4, pp. 677-695, 2010.

[3] D. Blömker, Amplitude Equations for Stochastic Partial Differential Equations, vol. 3 of Interdisciplinary Mathematical Sciences, World Scientific Publishing co.. Pte. Ltd., Hackensack, NJ, USA, 2007.

[4] G. Chen, J. Duan, and J. Zhang, "Geometric shape of invariant manifolds for a class of stochastic partial differential equations," Journal of Mathematical Physics, vol. 52, no. 7, 2011.

[5] M. D. Chekroun, H. Liu, and S. Wang, Approximation of Stochastic Invariant Manifolds, Springer Briefs in Mathematics, Springer-Verlag, New York, NY, USA, 2015.

[6] G. J. de Valcárcel, K. Staliunas, E. Roldán, and V. J. SánchezMorcillo, "Transverse patterns in degenerate optical parametric oscillation and degenerate four-wave mixing," Physical Review A: Atomic, Molecular and Optical Physics, vol. 54, no. 2, pp. 1609-1624, 1996.

[7] J. B. Swift and P. C. Hohenberg, "Hydrodynamic fluctuations at the convective instability," Physical Review A: Atomic, Molecular and Optical Physics, vol. 15, pp. 319-328, 1977.

[8] M. G. Clerc, A. Petrossian, and S. Residori, "Bouncing localized structures in a liquid-crystal light-valve experiment," Physical Review E: Statistical, Nonlinear, and Soft Matter Physics, vol. 71, no. 1, 2005.

[9] S. M. Cox, P. C. Matthews, and S. L. Pollicott, "Swift-Hohenberg model for magnetoconvection," Physical Review E: Statistical, Nonlinear, and Soft Matter Physics, vol. 69, no. 6, 2004.

[10] G. Kozyreff and M. Tlidi, "Nonvariational real Swift-Hohenberg equation for biological, chemical, and optical systems," Chaos: An Interdisciplinary Journal of Nonlinear Science, vol. 17, no. 3, 2007.

[11] M. Tlidi, P. Mandel, and R. Lefever, "Localized structures and localized patterns in optical bistability," Physical Review Letters, vol. 73, no. 5, pp. 640-643, 1994.

[12] K. Staliunas, "Laser Ginzburg-Landau equation and laser hydrodynamics," Physical Review A: Atomic, Molecular and Optical Physics, vol. 48, no. 2, pp. 1573-1581, 1993.

[13] J. Lega, J. V. Moloney, and A. C. Newell, "Swift-Hohenberg equation for lasers," Physical Review Letters, vol. 73, no. 22, pp. 2978-2981, 1994.

[14] K. Staliunas and V. J. Sánchez-Morcillo, "Transverse patterns in nonlinear optical resonators," Springer Verlag, Springer Tracts in Modern Physics, vol. 183, no. 13, 2003.

[15] P. C. Hohenberg and J. B. Swift, "Effects of additive noise at the onset of Rayleigh-Bénard convection," Physical Review A: Atomic, Molecular and Optical Physics, vol. 46, no. 8, pp. 47734785, 1992.

[16] Y. Pomeau and P. Manneville, "Wavelength selection in cellular flows," Physics Letters A, vol. 75, no. 4, pp. 296-298, 1980.

[17] J.-P. Eckmann and C. E. Wayne, "Propagating fronts and the center manifold theorem," Communications in Mathematical Physics, vol. 136, no. 2, pp. 285-307, 1991.

[18] M. F. Hilali, S. Métens, P. Borckmans, and G. Dewel, "Pattern selection in the generalized Swift-Hohenberg model," Physical 
Review E: Statistical, Nonlinear, and Soft Matter Physics, vol. 51, no. 3, pp. 2046-2052, 1995.

[19] D. Y. Hsieh, A. Q. Tang, and X. P. Wang, "On hydrodynamics instabilities, chaos, and phase transition," Acta Mechanica Sinica, vol. 12, pp. 1-14, 1996.

[20] A. Mielke and G. Schneider, "Attractors for modulation equations on unbounded domains-existence and comparison," Nonlinearity, vol. 8, no. 5, pp. 743-768, 1995.

[21] M. Polat, "Global attractor for a modified Swift-Hohenberg equation," Computers \& Mathematics with Applications, vol. 57, no. 1, pp. 62-66, 2009.

[22] C. Guo, Y. Guo, and C. Li, "Dynamic behaviors of a local modified stochastic Swift-Hohenberg equation with multiplicative noise," Boundary Value Problems, vol. 2017, no. 19, 13 pages, 2017.

[23] M. J. Capiński and P. Roldán, "Existence of a center manifold in a practical domain around L1 in the restricted three-body problem," SIAM Journal on Applied Dynamical Systems, vol. 11, no. 1, pp. 285-318, 2012.

[24] A. Pogan and K. Zumbrun, "Center manifolds for a class of degenerate evolution equations and existence of smallamplitude kinetic shocks," Journal of Differential Equations, vol. 264, no. 11, pp. 6752-6808, 2018. 


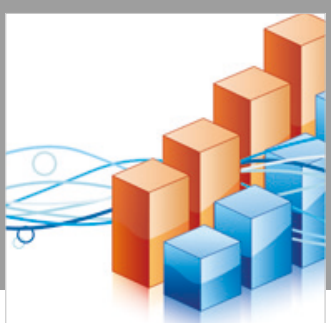

Advances in

Operations Research

\section{-n-m}
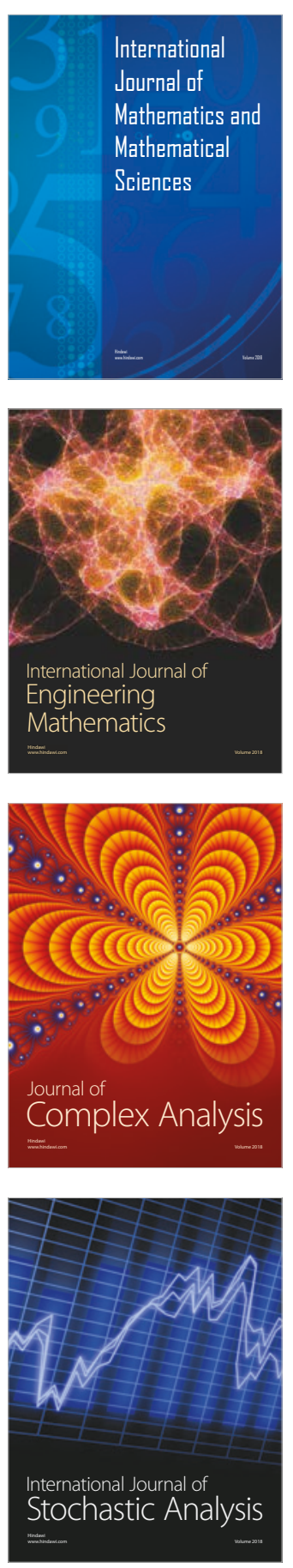
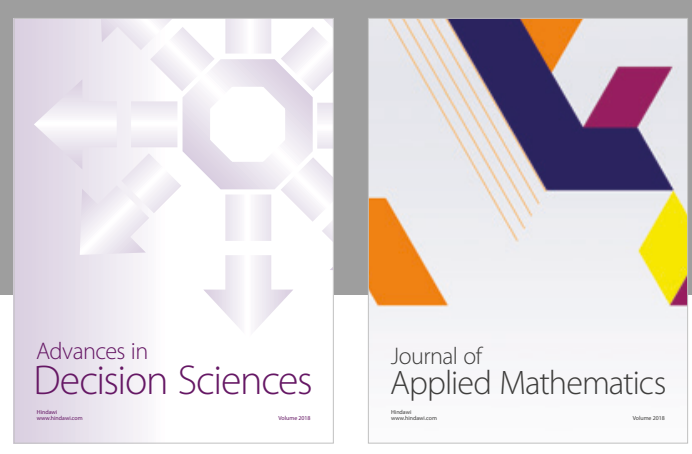

Journal of

Applied Mathematics
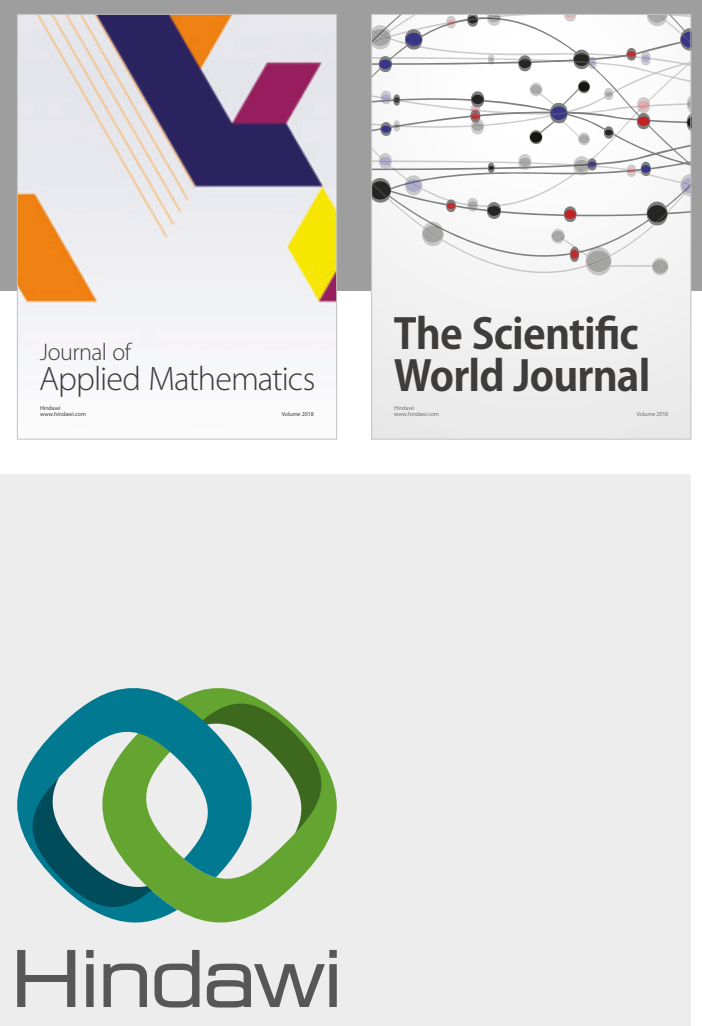

Submit your manuscripts at

www.hindawi.com

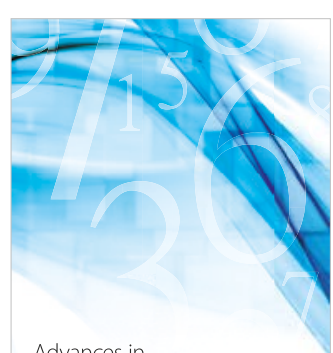

Advances in
Numerical Analysis
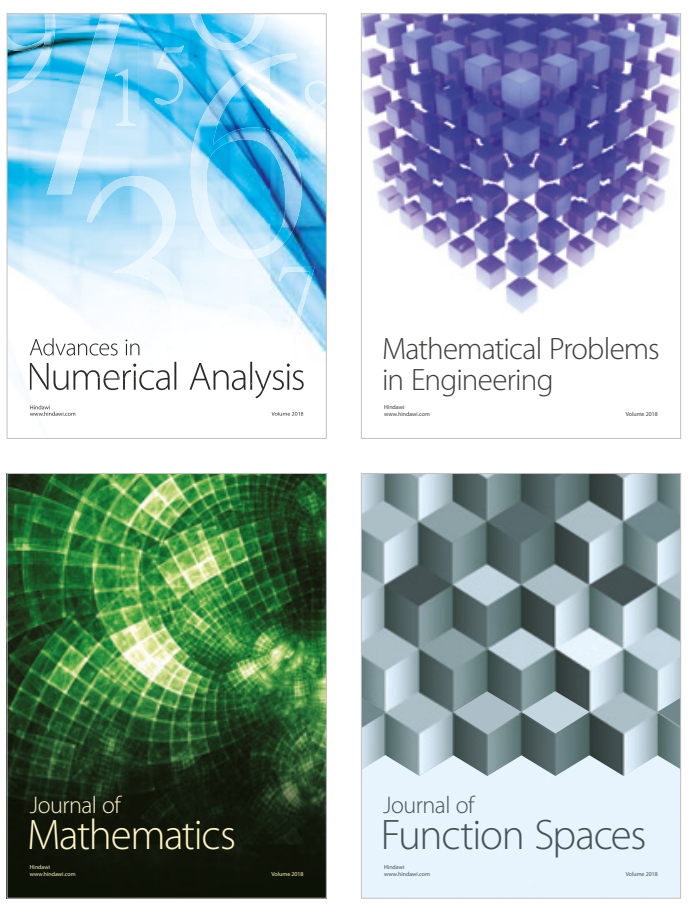

Mathematical Problems in Engineering

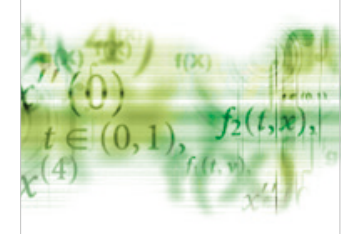

International Journal of

Differential Equations

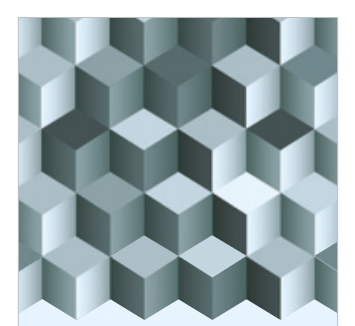

Journal of

Function Spaces

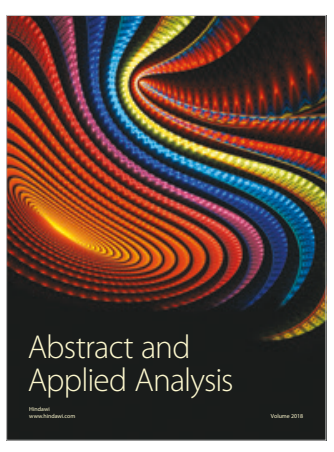

The Scientific

World Journal

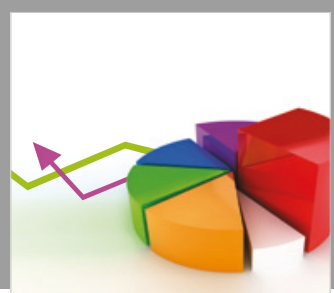

Journal of

Probability and Statistics
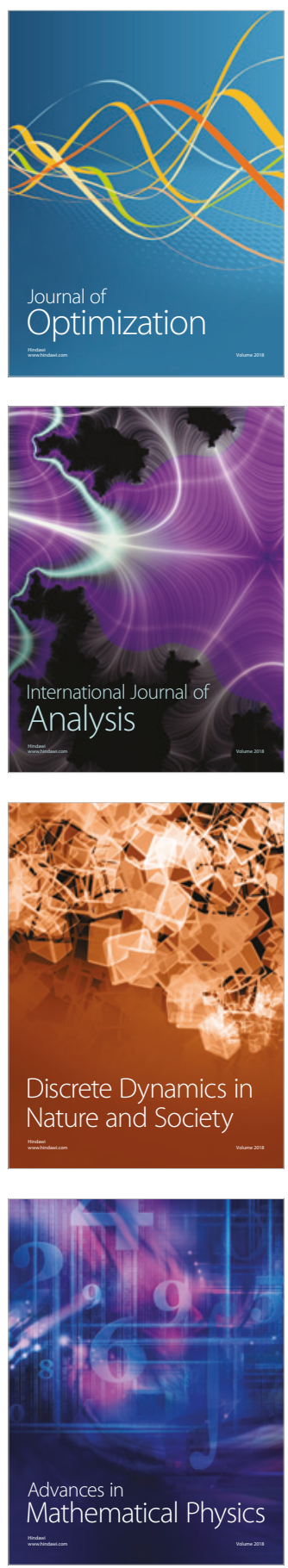